\title{
8. Das Uni-Zentrum Naturheilkunde an der Universitätsklinik Freiburg
}

ROMAN HUBER

Das Uni-Zentrum Naturheilkunde (UZN) bietet ein umfangreiches Behandlungsangebot im Bereich der Naturheilkunde und Komplementärmedizin mit den Versorgungsbereichen der

- konsiliarischen Behandlung am Universitätsklinikum Freiburg (jährlich ca. Goo Fälle)

- ambulanten Behandlung (Hochschulambulanz) mit jährlich > 4.00o Fällen

Das UZN ist dem Institute for Environmental Health Sciences der Universitätsklinik Freiburg zugeordnet.

Es werden akute und chronische Erkrankungen mit evaluierten Verfahren der Naturheilkunde und Komplementärmedizin (Anthroposophische Medizin, Akupunktur, etc.) in Kombination mit der konventionellen Methoden („Schulmedizin“) nach dem Konzept der Integrativen Medizin („Das Beste von beidem“) behandelt. Aus dem breiten Spektrum der Naturheilkunde werden in einem individuellen und wissenschaftlich geleiteten Ansatz die jeweils wirksamsten Therapieverfahren zusammengestellt (multimodale bzw. multidisziplinäre Komplextherapie). Hierbei wird auch gezielt versucht, die Selbstheilungskräfte des erkrankten Organismus anzuregen und einen gesundheitsfördernden Lebensstil sowie die Selbstwirksamkeit und salutogenetischen Ressourcen beim Erkrankten zu unterstützen. 


\section{Schwerpunktindikationen für die konsiliarische Behandlung sind:}

- Onkologische Erkrankungen (Abt. Gastroenterologie, Onkologie, Palliativmedizin des Universitätsklinikums Freiburg). Adjuvante Therapie von Krebserkrankungen (Integrative Onkologie)

- Psychosomatische Erkrankungen (Abt. Psychotherapie und Psychosomatische Medizin der Universitätsklinik Freiburg)

\section{Schwerpunktindikationen für die ambulante Behandlung sind:}

- Onkologische Erkrankungen

- Erschöpfungssyndrome, Burn-out, reaktive Depressionen

- Funktionelle und chronisch-entzündliche Magen-Darmerkrankungen (Reizdarm, M. Crohn, Colitis)

- Allergien

- Chronische Lebererkrankungen (chron. Hepatitis C, B, Leberzirrhose, seltene Lebererkrankungen)

- Bluthochdruck und chronische Herz-Kreislauferkrankungen

- Rheumatische Erkrankungen (Arthrosen, chronische Polyarthritis, Autoimmunerkrankungen)

- Hauterkrankungen wie Neurodermitis und Schuppenflechte

\subsection{Die diagnostischen Verfahren}

Das UZN verfügt über ein hochauflösendes Sonographiegerät, Lungenfunktion/Bodyplethysmographie, 12-Kanal EKG und Gerät zur Messung der Herzfrequenzvariabilität. Sämtliche anerkannten bildgebenden Verfahren und Laboruntersuchungen können am Universitätsklinikum Freiburg durchgeführt werden. Im UZN-eigenen Forschungslabor können darüber hinaus spezielle immunologische Untersuchungen durchgeführt werden.

Die spezielle naturheilkundliche Diagnostik umfasst verschieden Techniken der Erhebung von manuellen und reflektorischen Befunden, als auch von konstitutionellen Aspekten der traditionellen Europäischen und Chinesischen Medizin.

\subsection{Die therapeutischen Verfahren}

Es wird ein breites Spektrum von wissenschaftlich fundierten oder empirisch plausiblen Behandlungsverfahren der klassischen Naturheilverfahren und Komplementärmedizin angewendet.

Darüber hinaus kommen die Verfahren der Physikalischen Therapie zum Einsatz. Zudem besteht eine gute Zusammenarbeit mit niedergelassenen Therapeuten im Bereich Entspannungsverfahren, Kunsttherapie und manueller Therapie. 
Das Therapieangebot umfasst:

- Phytotherapie

- Ernährungstherapie und therapeutisches Fasten („Heilfasten“)

- Anthroposophische Medizin

- Akupunktur und Traditionelle Chinesische Medizin

- Mikrobiologische Therapie

- Lokoregionäre Radiofrequenz-Hyperthermie

- Traditionelle Verfahren: Blutegel, Schröpfen, Aderlaß

- Ordnungstherapie und Mind-Body-Medizin (Stressbewältigung, Entspannungsverfahren)

- Neuraltherapie und therapeutische Lokalanästhesie

- Manuelle Medizin

- Homöopathie

- Pflegerische Anwendungen in den Naturheilkunde (Auflagen, Einreibungen, mit Zusätzen)

- Hydrotherapie, Kneipptherapie, Wärme und Kälteanwendungen (Anleitung zur Selbst-Anwendung)

- Krankengymnastik und Physiotherapie, einschließlich verschiedener Formen der Massage (in Zusammenarbeit mit Therapeuten)

Am UZN arbeiten insgesamt 5 Ärztinnen und Ärzte, überwiegend mit langjähriger Erfahrung in Innere Medizin, Allgemeinmedizin und Naturheilkunde. Es bestehen u.a. Zusatzqualifikationen in Akupunktur, Anthroposophischer Medizin, Ernährungsmedizin, Homöopathie, manueller Medizin, Palliativmedizin, Psychotherapie und Psychosomatik, Physikalischer Therapie, Schmerztherapie, Umweltmedizin. Der Bereich der Ordnungstherapie/Mind-Body-Medizin wird durch niedergelassene Therapeutinnen und Therapeuten vertreten. In regelmäßigen Teamsitzungen werden Problempatienten besprochen und das individuelle Behandlungskonzept optimiert.

\subsection{Zuweisung und Kostenübernahme}

In der Hochschulambulanz können Patienten aller Kassen, nach Zuweisung durch Ihren behandelnden Arzt (Überweisungsschein) behandelt werden. Für Leistungen, die keine Kassenleistungen sind müssen die Patienten einen Selbstbehalt zahlen, der vom Zeitaufwand abhängt und nach GOÄ berechnet wird. 
IV Portraits einzelner Kliniken und Abteilungen

\subsection{Wissenschaft und Forschung}

Neben dem Angebot an klinischer Versorgung und medizinischen Leistungen ist das UZN ein wissenschaftliches klinisches Zentrum für Naturheilkunde mit einer umfangreichen Forschungsaktivität.

Schwerpunkt der Forschungsaktivitäten ist die Erforschung anthroposophischer Arzneimittel und pflanzlicher Präparate von der Grundlagenforschung an Zellkulturen bis hin zur Erforschung der Wirksamkeit in klinischen Studien.

Weitere Schwerpunkte der Forschungsaktivitäten ist das Themenfeld Fasten und Ernährungstherapie.

Es bestehen enge Kooperationen zu verschiedenen Abteilungen des Universitätsklinikums Freiburg sowie innerhalb des FORUMs universitärere naturheilkundlicher Einrichtungen im deutschsprachigen Raum. 